\title{
Deep learning (DL) Opens New Horizons in Personalized Medicine
}

\author{
Georgios Z. Papadakis*1,2 MD, MPH, PhD, Apostolos H. Karantanas ${ }^{1,2}$ MD, PhD and Kostas $\operatorname{Marias}^{1} \mathrm{PhD}^{*}$ \\ ${ }^{1}$ Computational Biomedicine Laboratory (CBML), Foundation for Research and Technology Hellas (FORTH), Heraklion, Crete, Greece \\ ${ }^{2}$ Department of Radiology-University of Crete and Department of Medical Imaging-Heraklion University Hospital, Greece
}

Received: 钢: December 05, 2018; Published: 跚: December 14, 2018

*Corresponding author: Georgios Z Papadakis MD, MPH, PhD, Computational Biomedicine Laboratory (CBML), Foundation for Research and Technology Hellas (FORTH), N. Plastira 100, Vassilika Vouton, GR-70013, Heraklion, Crete, Greece

\section{Opinion}

Although the idea of personalization of patient care dates to the time of Hippocrates, recent advances in diagnostic medical imaging and molecular medicine are gradually transforming health care services, by offering information and diagnostic tools enabling individualized patient management. One of the most critical challenges in personalized medicine is to transform big, multi-modal data into decision support tools, capable to bridge the translational gap to the clinical setting. Deep Learning (DL) provides a new approach to derive high-accuracy, multi-modal predictive models, which will enable implementation of the personalized medicine vision soon. Such models have led to unprecedented results, matching or even improving state-of-the-art prediction/detection rates based on both intuitive and non-intuitive disease descriptors. These results hold promise for significant socio- economic benefits from the application of DL personalized medicine. DL is a specialized machine learning approach based on multiple-layered structures (algorithms) of artificial neurons, which can process information and learn by adjusting the weights at each synapse, enabling performance of an intelligent task with high precision.

Furthermore, these deep networks can "learn" from big data and likewise human brain- generalize concepts and apply them to new data with high accuracy. This "intelligence" property has been expanded towards deep multimodal learning by integrating different sources of information (images, text, speech etc.) for further improving high-level multimodal decision support and reasoning [1]. As a result, current deep learning systems can integrate and model heterogeneous data from an individual patient, across modalities and time, allowing better predictions and recommending treatment options tailored to each patient's individual characteristics and needs. This concept is illustrated in Figure 1 highlighting the potential of deep learning to transform big healthcare data into useful tools for advancing personalized medicine. Reliable health predictors based on patient's heterogeneous Electronic Health Records (EHRs) data integration is another important application field of DL. In this direction, [2] developed the "Deep Patient" prediction system trained on 700,000 EHRs and demonstrated high disease prediction performance for severe diabetes, schizophrenia, and various malignancies [2]. Accurate diagnosis is a milestone for personalized medicine. Bejnordi, et al. [3] reported that 7 deep learning algorithms trained to detect metastases in hematoxylin and eosin-stained tissue sections of lymph nodes of women with breast cancer, outperformed a panel of 11 pathologists with an area under the curve of 0.994 (best algorithm) vs 0.884 (best pathologist) [3]. Madani, et al. [4] presented a DL echocardiography system for the automated diagnosis of cardiac disease to address the problem of echocardiographic assessment inaccuracy (up to 30\%) of echo reports, achieving 80\% accuracy in echocardiographic view classification and $92.3 \%$ accuracy for left ventricular hypertrophy classification [4].

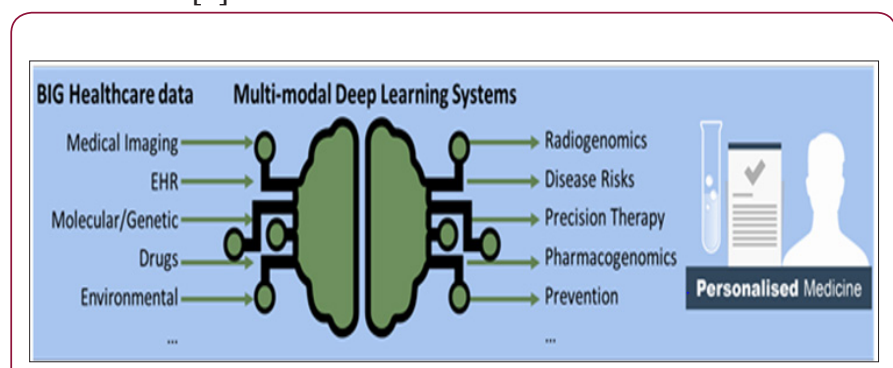

Figure 1: Multi-modal Deep Learning architectures can significantly contribute to the advancement of personalized medicine. 
Recently, Ding, et al. [5] proposed a DL system demonstrating improved early prediction of the final diagnosis of Alzheimer's Disease (AD) (82\% specificity at 100\% sensitivity, on average of 75.8 months prior to the final diagnosis) utilizing 18F- Fludeoxyglucose Positron Emission Tomography (PET) of the brain, and therefore enhancing opportunities for early therapeutic interventions [5]. DL has started revolutionizing decision support systems in Oncology due to the plethora of the available data and the heterogeneity of various malignancies, which require algorithms able to decipher hidden phenotype and genotype patterns and their associations. Recently [6] reported a deep learning system (NoduleX) which achieved an impressive accuracy (AUC $\sim 0.99$ ) in nodule malignancy classification ( $>1000$ nodules) based on CT scans, matching (or achieving?) thus the performance of experienced radiologists [6]. Previously, Zeju Li et al. had reported a DL brain tumor radiogenomic system able to predict with high accuracy the IDH1 mutation status solely from multi-modal Magnetic Resonance Imaging (MRI) data, achieving an impressive 95\% (?area under curve) AUC [7]. Using a similar approach, [8] demonstrated a deep learning system capable to predict the codeletion of $1 \mathrm{p} / 19 \mathrm{q}$ chromosome arms with high accuracy (87.7\%) in 159 low grade glioma patients [8]. In both cases, DL seems to enable the discovery of hidden imaging phenotype properties, which correlate with molecular/genetic information of high prognostic value.

In silico prediction of clinical outcome through therapy optimization, is of utmost importance for personalized medicine. Bibault, et al. [9] presented a DL Radiomics system able to predict complete response after neo-adjuvant chemoradiation for locally advanced rectal cancer with the goal to identify patients who would benefit more from conservative treatment rather than radical resections [9]. From a bioinformatics standpoint, [10] developed the Survival Net framework based on DL and Bayesian optimization for predicting clinical outcomes from big data, which was generated by diverse genomic platforms [10]. Drug discovery for personalization of therapy is another area that can benefit from the flexible architecture of DL systems towards de novo molecular design, reaction and bioactivity predictions [11]. In this direction IBM and Pfizer have joined forces for advancing immuno- oncology research with Watson for Drug Discovery [12]. Moreover, predicting environmental factors that influence health status is an integral part of preventive, personalized medicine. Ong et al. [13] proposed a time-series forecasting DL system for predicting the concentration of fine particulate matter $\mathrm{PM}_{2.5}$ (interfering with both human health and climate), in Japan, using exclusively real and publicly available sensor data, outperforming currently used methods [13].

Based on this data, DL architectures have already demonstrated significant technological advances across the personalized medicine informatics application spectrum, due to their versatility towards integrating multi-modal clinical data, discovering hidden properties and successfully generalizing results in unseen datasets. The number of DL applications for personalized medicine will continue to grow due to high demand of such technologies and their anticipated socioeconomic impact. Although this process is facilitated by the widely available DL open computational frameworks and the abundance of big healthcare data, there is still skepticism regarding the clinical adoption of DL technologies emanating from the lack of causality and their "black-box" nature. The inability to understand why an algorithm achieves generalization and performs so well, might be a critical factor inhibiting clinical translation of DL technologies. To address this problem, several researchers are focusing on explaining the inner organization of DL networks.

Tishby and co-workers have proposed an influential theory based on the "information bottleneck" concept, according to which the network compresses input data as if by squeezing the information through a bottleneck, retaining only the features most relevant to the general concepts of the learning task $[14,15]$. This compression process is pronounced at the higher layers of the network for promoting better predictions via preserving information relevant to the output labels at the expense of input data information, which is gradually "forgotten". The confirmation of such theoretical explanatory models in conjunction with big data validation across clinical sites and patient data registries, is a sine qua non condition for accelerating the clinical translation of DL personalized medicine decision support systems.

\section{References}

1. Noda K, Arie H, Suga Y, Ogata T (2014) Multimodal integration learning of robot behavior using deep neural networks. Rob \& Auton Syst 62(6): 721-736.

2. Miotto R, Li L, Kidd B, Dudley J (2016) Deep patient: An unsupervised representation to predict the future of patients from the electronic health Records. Sci Rep 17(6):26094.

3. Bejnordi B, Veta M, Van Diest P, Van Ginneken B, Karssemeijer N, et al. (2017) Diagnostic assessment of deep learning algorithms for detection of lymph node metastases in women with breast cancer. JAMA 318(22): 2199-2210.

4. Madani A, Ong JR, Tibrewal A, Mofrad MRK (2018) Deep echocardiography: data-efficient supervised and semi-supervised deep learning towards automated diagnosis of cardiac disease. npj Digital Medicine 1 (1): 59.

5. Ding Y, Sohn JH, Kawczynski MG, Trivedi H, Harnish R, et al. (2018) A A deep learning model to predict a diagnosis of Alzheimer disease by using 18F-FDG PET of the bain. Radiology 2018, doi: 10.1148/ radiol.2018180958.

6. Causey J, Zhang J, Ma S, Jiang B, Qualls J, et al. (2018) Highly accurate model for prediction of lung nodule malignancy with CT scans. Sci Rep 8(1): 9286.

7. Li Z, Wang Y, Yu J, Guo Y, Cao W (2017) Deep learning based radiomics (DLR) and its usage in noninvasive IDH1 prediction for low grade glioma. Sci Rep 7(1):5467.

8. Akkus Z, Ali I, Sedlar J, Agrawal JP, Parney IF, et al. (2017) Predicting deletion of chromosomal arms 1p/19q in low-grade gliomas from MR images using machine intelligence. J Digit Imaging 30(4): 469-476.

9. Bibault J, Giraud P, Durdux C, Taieb J, Berger A, et al. (2018) Deep learning and radiomics predict complete response after neo-adjuvant chemoradiation for locally advanced rectal cancer. Sci Rep 8(1):12611.

10. Yousefi S, Amrollahi F, Amgad M, Dong C, Lewis JE, et al. (2017) Predicting clinical outcomes from large scale cancer genomic profiles with deep survival models. Sci Rep 7(1): 11707. 
11. Chen H, Engkvist O, Wang Y, Olivecrona M, Blaschke T, et al. (2018) The rise of deep learning in drug discovery. Drug Discovery Today 23(6): $1241-1250$

12. Pfizer:https://www.pfizer.com/news/press-release/press-release detail/ibm_and_pfizer_to_accelerate_immuno_oncology_research_with_ watson_for_drug_disco very

13. Ong BT, Sugiura K, Zettsu K (2016) Dynamically pre-trained deep recurrent neural networks using environmental monitoring data for predicting $\mathrm{PM}_{2.5}$. Neural Comput \& Applic 27(6): 1553-1566.

\section{ISSN: 2574-1241}

DOI: 10.26717/BJSTR.2018.12.002215

Georgios Z Papadakis. Biomed J Sci \& Tech Res

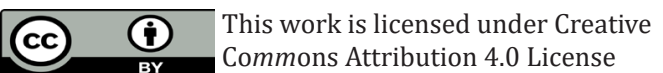

Submission Link: https://biomedres.us/submit-manuscript.php
14. Shwartz Ziv R, Tishby N (2017) Opening the black box of deep neural networks via information. arXiv preprint.

15. Tishby N, Zaslavsky N (2015) Deep learning and the information bottleneck principle. In 2015 IEEE Information Theory Workshop: pp. $1-5$.

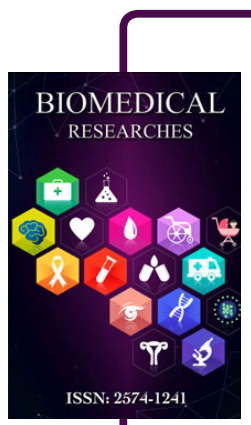

Assets of Publishing with us

- Global archiving of articles

- Immediate, unrestricted online access

- Rigorous Peer Review Process

- Authors Retain Copyrights

- Unique DOI for all articles

https://biomedres.us/ 